\title{
Severe Obliterative Bronchitis Associated with Stevens-Johnson Syndrome
}

\author{
Tetsukan Woo ${ }^{1}$, Haruhiro Saito ${ }^{1}$, Yasushi Yamakawa ${ }^{1}$, Shigeru Komatsu ${ }^{1}$, Sumi Onuma ${ }^{2}$, \\ Koji Okudela ${ }^{3}$, Akinori Nozawa ${ }^{4}$, Michiko Aihara ${ }^{2}$, \\ Zenro Ikezawa $^{2}$ and Yoshiaki Ishigatsubo ${ }^{1}$
}

\begin{abstract}
We report a case of Stevens-Johnson syndrome (SJS) in which the patient had been diagnosed with severe obliterative bronchitis. A 29-year-old woman was admitted with a high fever and a widespread vesicular rash. She was diagnosed with SJS and betamethasone administration was started. After one month, her vesicular skin rash improved; however, she developed respiratory failure and was assisted with mechanical ventilation. Computed tomography of the chest demonstrated a hyperlucent lung with narrowing of the peripheral vessels. Bronchoscopy revealed an occlusion of the bronchus when the patient exhaled. The flow-volume curve revealed a severe obstructive pattern. The patient was diagnosed with obliterative bronchitis following SJS. She was treated with a bronchodilator and steroids, but could not breathe adequately without the ventilator. During the following year, her $\mathrm{PaCO}_{2}$ increased to 100 torr and her heart function also continued to worsen. Despite intensive treatment, she died one year and seven months after the onset of SJS. In SJS and toxic epidermal necrolysis (TEN) patients, chronic pulmonary complications are rare, but there is no effective therapy for obliterative bronchitis following SJS/TEN. Therefore, early awareness of this condition is needed and lung transplantation must be considered at an early stage of this disease.
\end{abstract}

Key words: obliterative bronchitis, Stevens-Johnson syndrome

(Intern Med 50: 2823-2827, 2011)

(DOI: 10.2169/internalmedicine.50.5582)

\section{Introduction}

Stevens-Johnson syndrome (SJS) and toxic epidermal necrolysis (TEN) are rare but severe diseases characterized by extensive detachment of the epidermis and mucous membrane involvement (1). It is generally accepted that SJS/TEN are usually adverse drug reactions, although various etiologic factors have been implicated as causes of SJS/TEN including infection, vaccination, drugs and systemic diseases $(2,3)$. The mortality rate is reported to be between 1 $5 \%$ for SJS and $10-40 \%$ for TEN $(4,5)$.

SJS and TEN present as a febrile illness associated with erythematous macules and atypical target lesions followed by skin detachment. Involvement of ocular, oral and genital mucosa occurs in both illnesses. SJS and TEN are currently considered to be a part of a spectrum of clinically and pathologically related diseases (1-3). Within this spectrum, TEN is the most severe type with epidermal detachment involving more than $30 \%$ of total body surface area (TBSA), while SJS is a milder type with less epidermal detachment (1). The pathogenesis of SJS/TEN is not understood, but it is well known that the basic epidermal and mucosal pathology in SJS/TEN is large-scale epidermal and mucosal death, which is the result of apoptosis (7).

SJS and TEN are often accompanied by visceral involvement, including pulmonary complications, hepatitis, gastrointestinal hemorrhage, and glomerulonephritis (7-10). Ac-

\footnotetext{
${ }^{1}$ Department of Respiratory Medicine, Yokohama City University School of Medicine, Japan, ${ }^{2}$ Department of Dermatology, Yokohama City University School of Medicine, Japan, ${ }^{3}$ Department of Pathology, Yokohama City University School of Medicine, Japan and ${ }^{4}$ Department of Pathology, Yokohama City University Medical Center, Japan

Received for publication March 30, 2011; Accepted for publication August 5, 2011

Correspondence to Dr. Tetsukan Woo, tetsu.n.u@ cotton.ocn.ne.jp
} 

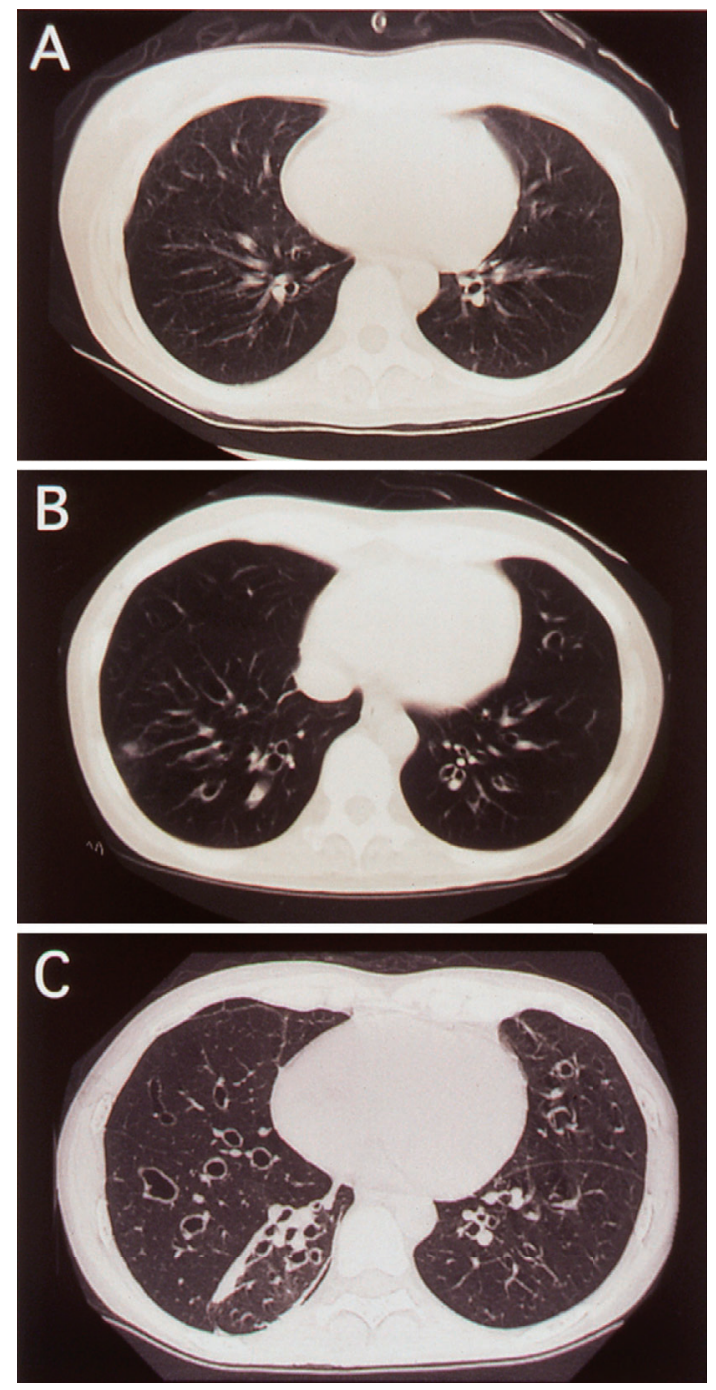

Figure 1. A computed tomography of the chest demonstrated a hyperlucent lung with narrowing of the peripheral vessels on the 27th day (A). Bronchiectasis and atelectasis were observed in the right lower lung on the 189th day (B). Advanced bronchiectasis and atelectasis were observed on the 268th day (C).

cording to previous reports, pulmonary complications associated with SJS/TEN have been divided into two types: interstitial pneumonia in the acute phase and obliterative bronchitis or bronchiolitis in the chronic phase (9). However, there are only a few reports concerning chronic pulmonary complications in patients with SJS/TEN (11-23). We herein report a patient with SJS who had been diagnosed with severe obliterative bronchitis and died due to respiratory failure.

\section{Case Report}

A 29-year-old woman was admitted with a high fever and a widespread vesicular rash associated with erosions of the oral mucosa, conjunctivitis and laryngitis. She had been taking an antipyretic drug containing acetaminophen for several days for a slight fever and cough. She had no history of al- lergic diseases or other previous illnesses.

On admission, epidermal detachment involved less than $10 \%$ of TBSA. Laboratory tests were normal except for the following: Blood data showed a WBC of $8,100 / \mu \mathrm{L}$ (band.3.0\%, seg.77.5\%, mono.12.5\%, lym.6.5\%), CRP 1.7 $\mathrm{mg} / \mathrm{dL}$, and liver dysfunction was observed (ALT $114 \mathrm{mU} /$ $\mathrm{mL}$, AST $657 \mathrm{mU} / \mathrm{mL}$, LDH $600 \mathrm{mU} / \mathrm{mL}$ ). Mycoplasma and chlamydia infections and other viral infections (herpes simplex virus, cytomegalovirus) were negative on serological tests.

Under a diagnosis of SJS, administration of betamethasone was started at $10 \mathrm{mg} /$ day. The patient began to improve about one week after starting the treatment and betamethasone was reduced gradually over one month. However, she developed progressive dyspnea attacks, with wheezing and a respiratory rate of $24 / \mathrm{min}$. Wheezing was audible in the bilateral chest, the oral mucosa was slightly dry, her skin was pigmented and conjunctival adhesion was observed.

An arterial blood gas examination performed on the 27th day of hospitalization revealed $\mathrm{pH}$ of $7.390, \mathrm{PaCO}_{2}$ of 62.3 torr and $\mathrm{PaO}_{2}$ of 88.0 torr during $\mathrm{FiO}_{2}$ of 0.6. Sputum culture revealed methicillin-resistant Staphylococcus aureus (MRSA). Chest CT showed bilateral hyperlucencies with narrowing of the peripheral vessels (Fig. 1A). The dyspnea attack was accompanied by wheezing and heavy sputum secretion.

The first time, her symptoms were thought to be caused by acute bronchitis. She was treated with intravenous aminophylline, a $\beta_{2}$-stimulant inhaler and betamethasone 6 $\mathrm{mg}$ /day. Oral clarythromycin was also administered from her 61st day of hospitalization. However, her dyspnea worsened and $\mathrm{PaCO}_{2}$ elevated to 100 torr. She developed $\mathrm{CO}_{2}$ narcosis and required mechanical ventilation support on her 80th day of hospitalization. Her chest X-ray did not show any obvious abnormalities without hyperlucencies. Mechanical ventilation support was used with pressure support and endexpiratory pressure (PEEP). Intravenous aminophylline and prednisolone were administered with a $\beta_{2}$-stimulant inhaler, beclomethasone inhaler, and antibiotics. With the ventilation support, her $\mathrm{PaCO}_{2}$ improved to 60 torr, and we tried several times to wean her from the ventilator, but failed. We concluded that weaning was impossible and mechanical ventilation was continued.

Bronchoscopy performed on the 86th day of hospitalization revealed an occlusion of the fifth bronchus on exhalation (Fig. 2). A pulmonary function test was performed on the 104th day of hospitalization. The flow-volume curve revealed a severe obstructive pattern, suggesting the presence of an obstruction in the relatively central bronchus (Fig. 3). A biopsy of the bronchial mucosa was performed on the 86th day of hospitalization. Biopsied tissues from the right second carina showed squamous metaplasia (Fig. 4A), goblet cell hyperplasia and submucosal edema (Fig. 4B). These findings suggested remodeling of the bronchial membrane after severe erosive bronchitis as a result of SJS. Sputum 

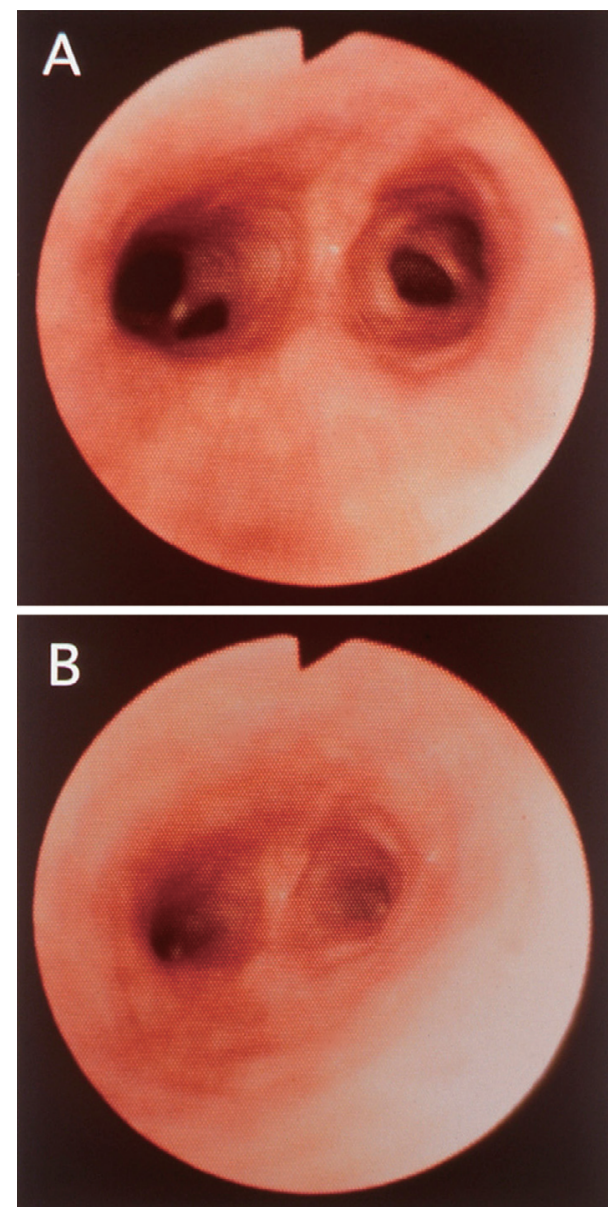

Figure 2. View of the right B8a by bronchofibroscopy on the 86th day. The upper photo (A) showed the bronchus on inspiration. The lower photo (B) showed the bronchus on expiration. These findings showed that the bronchus was completely obstructed on expiration.

culture was examined once a week, and it revealed MRSA intermittently.

Chest CT showed bronchiectasis and atelectasis (Fig. 1B). The atelectasis was improved by bronchial lavage, and many mucus plugs were observed in the lavage fluid. Finally, she was diagnosed with obliterative bronchitis following SJS. Despite all the medical treatment in combination with the mechanical ventilator, her $\mathrm{PaCO}_{2}$ retention increased gradually, accompanied by heavy discharge requiring frequent suction. Chest CT showed advanced bronchiectasis and atelectasis (Fig. 1C). She suffered from repeated pneumothorax and pneumomediastinum during her clinical course, and she became emotionally disturbed and needed antidepressant drugs. Lung transplantation was considered the best treatment option; however, since she had undergone mechanical ventilation for several months and her general and mental conditions were poor, the lung transplant option was rejected.

One year after the onset of SIS, the patient continued on the mechanical ventilator, and her $\mathrm{PaCO}_{2}$ was about 100 140 torr at pressure control ventilation of $28 \mathrm{mmH}_{2} \mathrm{O}$ with PEEP of $6 \mathrm{cmH}_{2} \mathrm{O}$. Her heart function also continued to

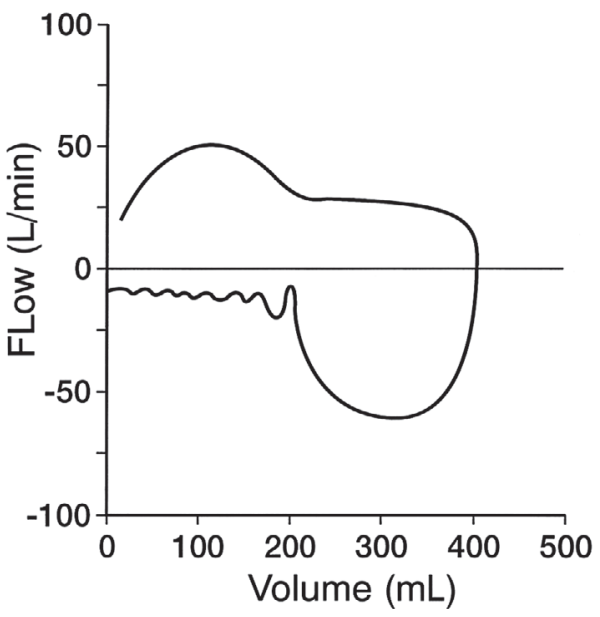

Figure 3. The flow-volume curve showed air flow limitation and air trapping on expiration under conditions of PS $8 \mathrm{~cm}-$ $\mathrm{H}_{2} \mathrm{O}$ and PEEP $5 \mathrm{cmH}_{2} \mathrm{O}$ on the 104th day, suggesting the presence of an obstruction in the relatively central bronchus.

worsen. Moreover, she suffered from bilateral pneumothorax, and underwent bilateral thoracic drainage. Despite the intensive treatment, she died one year and seven months after the onset of SJS.

\section{Discussion}

The present patient experienced severe respiratory failure as a result of obliterative bronchitis following SJS. Pulmonary complications in the chronic phase of SJS/TEN are rare, and most of them are obliterative bronchitis or bronchiolitis (9). Fourteen cases of obliterative bronchitis or bronchiolitis following SJS/TEN have been reported (11-23). Two cases were thought to result from SJS brought on by mycoplasma infections $(11,12)$, and 11 cases were thought to have been related to SJS/TEN caused by drugs (13-19, 21-23). Six cases of severe respiratory failure, similar to that of the present patient, resulted in fatalities from 2 months to one and a half years after the onset of SJS/TEN $(11,12,15,20,21,23)$. One patient developed progressive respiratory failure due to obliterative bronchiolitis, and underwent a living-donor lung transplantation 6 months after the onset of SJS (22). The other patients were discharged, but they all had prolonged respiratory complaints $(13,14,16-19)$. There are no reports of patients who have survived on mechanical ventilation due to obliterative bronchitis or bronchiolitis after SJS / TEN $(11,12,15,20,21,23)$.

The pathogenesis of SJS/TEN and its pulmonary complications are not well understood. It has been suggested that several pathways are implicated in the widespread apoptosis of keratinocytes in SJS/TEN (24-27). Involvement of cytotoxic $\mathrm{T}$ cells and the molecular cytotoxicity of Fas and cytotoxic enzymes, including granzyme $\mathrm{B}$, perforin, and granulysin have been shown in SJS/TEN $(25,27)$. Cytokines including tumor necrosis factor alpha $(\mathrm{TNF}-\alpha)$ and inter- 


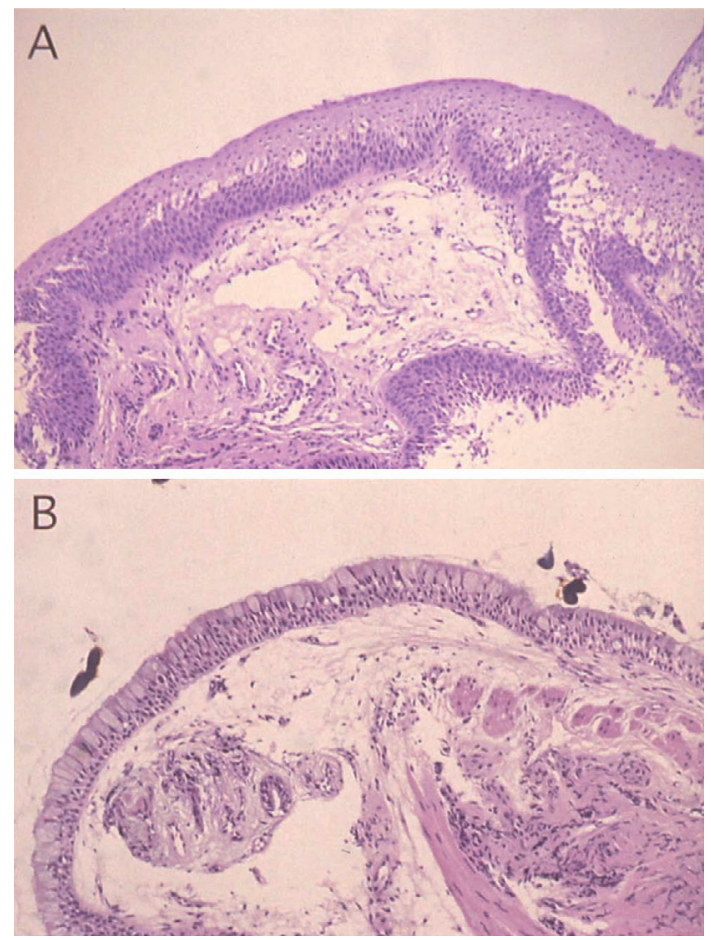

Figure 4. Biopsied tissues from the right second carina showed squamous metaplasia (A), goblet cell hyperplasia and submucosal edema (B) on the 86th day (Hematoxylin and Eosin staining, $\times 100$ ). These findings suggested remodeling of the bronchial mucosa after severe erosive bronchitis as a result of Stevens-Johnson syndrome.

feron gamma (IFN- $\gamma$ ) were found to be overexpressed in the lesional skin $(24,25,28,29)$. Wuepper et al demonstrated that immunocomplexes were detected in the serum and dermal/mucosal lesions of severely affected SJS patients (30), and those immunocomplexes were thought to be a possible cause of chronic pulmonary complications after SJS/ TEN (23).

Secondary infections are also thought to be another cause of late pulmonary deterioration. In fact, MRSA was detected in the present patient's sputum culture. Although her infection was controlled by antibiotics, the secondary infection was thought to be a possible cause of her bronchitis.

In the present case, we assume that the remodeling of the bronchial mucosa in the form of fibrosis and scarring was the result of severe inflammation due to SJS, and also a cause of the obstructive changes. In fact, biopsied tissues from the bronchial mucosa revealed that there was little infiltration of inflammatory cells, while remodeling of the bronchial epithelium was observed. Two autopsy cases with obliterative bronchitis and bronchiolitis after SJS also showed loss of the bronchial epithelium, while the luminal space was totally replaced by fibrous granulation tissue $(12,15)$.

We think that the etiologic factor of SJS in our case was an antipyretic drug ingested before admission, although her drug-induced lymphocyte stimulation test (DLST) for traces of the drug using peripheral blood mononuclear cells was negative. We cannot rule out the possibility that an infectious agent that we could not detect may have induced SJS, but the clinical course strongly suggests the antipyretic medicine was the etiologic factor. Yetiv et al reported that an allergic predisposition might have played a role in the development of SJS, and drugs and infections were especially suspect as etiologic agents in SJS (31).

Once the obstructive changes have been established, there is currently no curative therapy for obliterative bronchitis and bronchiolitis $(32,33)$. Steroids have been reported for the treatment of these conditions $(15,18,23)$, but they only mitigate bronchial edema and improve the symptoms temporarily $(15,18,23)$. In the present case, intravenous prednisolone and a beclomethasone inhaler were thought to be effective in reducing the bronchial mucosa edema and secretion to some degree. Our patient's breathing was disrupted by heavy bronchial discharge, and the use of influent suction and squeezing was effective in excreting the patient's sputum. Immunosuppressants such as cyclosporine are also used for the treatment of obliterative bronchiolitis, but the effect is uncertain $(33,34)$.

Lung transplantation is thought to be the only curative therapy for such a case (22). However, patients using mechanical ventilation for a long time, who have other complications and who cannot achieve total rehabilitation, are not candidates for lung transplantation. In SJS/TEN patients, chronic pulmonary complications are rare, but there is still no effective therapy for obliterative bronchitis and bronchiolitis following SJS/TEN. Therefore, early awareness of this condition is needed and lung transplantation must be considered at an early stage of this disease.

The authors state that they have no Conflict of Interest (COI).

\section{References}

1. Bastuji-Garin S, Rzany B, Stern RS, Shear NH, Naldi L, Roujeau JC. Clinical classification of cases of toxic epidermal necrolysis, Stevens-Johnson syndrome, and erythema multiforme. Arch Dermatol 129: 92-96, 1993.

2. Letko E, Papaliodis DN, Papaliodis GN, Daoud YJ, Ahmed AR, Foster CS. Stevens-Johnson syndrome and toxic epidermal necrolysis: a review of the literature. Ann Allergy Asthma Immunol 94: 419-436, 2005.

3. Pereira FA, Mudgil AV, Rosmarin DM. Toxic epidermal necrolysis. J Am Acad Dermatol 56: 181-200, 2007.

4. Schneck J, Fagot JP, Sekula P, Sassolas B, Roujeau JC, Mockenhaupt M. Effects of treatments on the mortality of StevensJohnson syndrome and toxic epidermal necrolysis: A retrospective study on patients included in the prospective Euro SCAR Study. J Am Acad Dermatol 58: 33-40, 2008.

5. Roujeau JC, Stern RS. Severe adverse cutaneous reactions to drugs. N Engl J Med 331: 1272-1285, 1994.

6. Tripathi A, Ditto AM, Grammer LC, et al. Corticosteroid therapy in an additional 13 cases of Stevens-Johnson syndrome: a total series of 67 cases. Allergy and Asthma Proc 21: 101-105, 2000.

7. Chave TA, Mortimer NJ, Sladden MJ, Hall AP, Hutchinson PE. Toxic epidermal necrolysis: current evedence, practical management and future directions. Br J Dermatol 153: 241-253, 2005. 
8. Schöpf E, Stühmer A, Rzany B, Victor N, Zentgraf R, Kapp JF. Toxic epidermal necrolysis and Stevens-Johnson syndrome: an epidemiologic study from West Germany. Arch Dermatol 127: 839-842, 1991.

9. Kamata T, Sakamaki F, Fujita H, Urano T, Mori M. Toxic epidermal necrolysis with tracheobronchial and pulmonary complications. Intern Med 33: 252-255, 1994.

10. Schamberger MS, Goel J, Braddock SR, Parsons DS, Tobias JD. Stevens-Johnson syndrome and respiratory failure in a 9-year-old boy. Southern Med J 90: 755-757, 1997.

11. Schønheyder H. Stevens-Johnson syndrome associated with intrahepatic cholestasis and respiratory disease: a case report. Acta Derm Venereol 61: 171-173, 1981.

12. Edwards C, Penny M, Newman J. Mycoplasma pneumonia Stevens-Johnson syndrome, and chronic obliterative bronchitis. Thorax 38: 867-869, 1983.

13. Virant FS, Redding GJ, Novack AH. Multiple pulmonary complications in a patient with Stevens-Johnson syndrome. Clin Pediatr (Phila) 23: 412-414, 1984.

14. Reyes de la Rocha S, Leonard JC, Demetriou E. Potential permanent respiratory sequela of Stevens-Johnson syndrome in an adolescent. J Adolesc Health Care 6: 220-223, 1985.

15. Tsunoda N, Iwanaga T, Saito T, Kitamura S, Saito K. Rapidly progressive bronchiolitis obliterans associated with StevensJohnson syndrome. Chest 98: 243-245, 1990.

16. Martín Mateos MA, Polemeque A, Pastor X, Muñoz López F. Uncommon serious complications in Stevens-Johnson syndrome: a clinical case. J Investig Allergol Clin Immunol 2: 278-283, 1992.

17. Edell DS, Davidson JJ, Muelenaer AA, Majure M. Unusual manifestation of Stevens-Johnson syndrome involving the respiratory and gastrointestinal tract. Pediatrics 89: 429-432, 1992.

18. Yatsunami J, Nakanishi Y, Matsuki H, et al. Chronic bronchobronchiolitis obliterans associated with Stevens-Johnson syndrome. Intern Med 34: 772-775, 1995.

19. Kim MJ, Lee KY. Bronchiolitis obliterans in children with Stevens-Johnson syndrome: follow-up with high resolution CT. Pediatr Radiol 26: 22-25, 1996.

20. McIvor RA, Zaidi J, Peters WJ, Hyland RH. Acute and chronic respiratory complications of toxic epidermal necrolysis. J Burn Care Rehabil 17: 237-240, 1996.

21. Minamihaba O, Nakamura H, Sata M, et al. Progressive bronchial obstruction associated with toxic epidermal necrolysis. Respirol- ogy 4: 93-95, 1999.

22. Date H, Sano Y, Aoe M, et al. Living-donor lobar lung transplantation for bronchiolitis obliterans after Stevens-Johnson syndrome. J Thorac Cardiovasc Surg 123: 389-391, 2002.

23. Kamada N, Kinoshita K, Togawa $\mathrm{Y}$, et al. Chronic pulmonary complications associated with toxic epidermal necrolysis: report of a severe case with anti-Ro/SS-A and a review of the published work. J Dermatol 33: 616-622, 2006.

24. Paquet P, Nikkels A, Arrese JE, Vanderkelen A, Pierard GE. Macrophages and tumor necrosis factor alpha in toxic epidermal necrolysis. Arch Dermatol 130: 605-608, 1997.

25. Chung WH, Hung SI, Yang JY, et al. Granulysin is a key mediator for disseminated keratinocyte death in Stevens-Johnson syndrome and toxic epidermal necrolysis. Nat Med 14: 1343-1350, 2008.

26. Konur A, Schulz U, Eissner G, Andreesen R, Holler E. Interferon (IFN)-gamma is a main mediator of keratinocyte (HaCaT) apoptosis and contributes to autocrine IFN-gamma and tumour necrosis factor-alpha production. Br J Dermatol 152: 1134-1142, 2005.

27. Viard I, Wehrli P, Bullani R, et al. Inhibition of toxic epidermal necrolysis by blockade of CD95 with human intravenous immunoglobulin. Science 282: 490-493, 1998.

28. Rhodes LE, Hashim IA, MacLaughlin PJ, Friedmann PS. Blister fluid cytokines in cutaneous inflammatory bullous disorders. Acta Derm venereal 79: 288-290, 1999.

29. Nassif A, Moslehi H, Le Gouvello S, et al. Evaluation of the potential role of cytokines in toxic epidermal necrolysis. J Invest Dermatol 123: 850-855, 2004.

30. Wuepper KD, Watson PA, Kazmierowski JA. Immunecomplexes in erythema multiforme and the Stevens-Johnson syndrome. J Invest Dermatol 74: 368-371, 1980.

31. Yetiv JZ, Bianchine JR, Owen JA Jr. Etiologic factors of StevensJohnson syndrome. Southern Med J 73: 599-602, 1980.

32. Yokoi T, Hirabayashi N, Ito M, Uno U, Tsuzuki T. Bronchobronchitis oblitarans as a complication of bone marrow transplantation: clinicopathological study of eight autopsy study. Virchowa Arch 431: 275-282, 1997.

33. Palmas A, Tefferi A, Myers JL, et al. Late-onset noninfectious pulmonary complications after allogeneic bone marrow transplantation. Br J Haematol 100: 680-687, 1998.

34. Khurshid I, Anderson LC. Non-infectious pulmonary complications after bone marrow transplantation. Postgrad Med J 78: 257262, 2002.

(C) 2011 The Japanese Society of Internal Medicine http://www.naika.or.jp/imindex.html 